\title{
Research on Data Conversion and Integration Method of Assembly Process System
}

\author{
Hu HAN \\ College of Mechanical Engineering, Linyi University, Linyi 276005, Shandong, China \\ Huanfang MA \\ Linyi New Tian Li Machinary Co.,Ltd
}

\begin{abstract}
According to the characteristics of assembly process data, through secondary development of CAD system, centering on the data model of the aircraft assembly process, PPR data model, the hierarchal structure data model, is proposed and the conversion among various types of assembly process data model are implemented, the basis for following tasks of digital assembly is provided; at the same time, the assembly process system is developed, and the system can be integrated with PDM system. At Last, an example of assembly on the components of aircraft is given, and the result is proved to be effective.
\end{abstract}

KEYWORD: Data Conversion; Data Integration; Assembly Process System; PPR

\section{GENERAL INSTRUCTIONS}

Data conversion technology from the CAD system to the assembly environment has already been studied by many people at home and abroad. These researches combined with virtual assembly, can be mainly divided into two types: 1)Making use of neutral file(Initial Graphics exchange Specification (IGS), STandard for the exchange of Product model data (STP), STereo Lithography interface specification (STL) or virtual reality modeling language (VRML), etc.), VADE system uses STP as a medium to transmit information[1]. This method is simple and straightforward but there are obvious shortcomings, they are: first, to the neutral file, sometimes the models are simplified and approximate, some information, especially required information is easily lost; second, and sometimes the models contain more redundant information, data conversion efficiency is not high. (2)Making use of CAD system developed by temselves, the CAD design system and virtual assembly system together share the underlying database, such as: Concept Dymamics, VAVAS from Zhejing University[2]. These methods can be implemented in system assembly modeling and virtual assembly without data conversion, but it is difficult to integrate with mainstream CAD systems.

Traditional assembly models are mainly: relation model[3], hierarchical model and the virtual link model[4]. These models are proposed on the basis of traditional CAD environment based on WIMP interface. However the operating of WIMP interface environment is process-oriented, so, they contain only static structures representation information without reflecting the interaction of the dynamic assembly process. At present, because the direct operation and multi-channel interactive approach in virtual assembly system is task-oriented, the corresponding assembly model should not only describe the static structure, but also reflect the dynamic process of assembly interactions [5-9]. Centering on the data model of the assembly process, a hierarchy data model based on PPR is proposed and the conversion among various types of aircraft assembly process data model are implemented, the basis for following tasks of digital assembly of aircraft is provided.

\section{DATA TRANSFERRING PROCESS BETWEEN ASSEMBLY SYSTEM}

The system takes the assembly process design of C919 type aircraft as an example. The workflow is shown in Figure 1, Digital assembly process system obtains product engineering data (EBOM data) and 3D digital model from enterprise PDM system; through data reception module, imports product engineering data (EBOM data) and 3D digital model into digital assembly system, through the visualization process planning module, divides product engineering data (EBOM data) into the initial product manufacturing data (MBOM data) and generates process structure data. On the basis of process structure data, through detailed process design module and process simulation module, process engineers generate $3 \mathrm{D} \mathrm{AO}$ document to 
guide product assembly, and through the file upload module, uploads them to enterprise PDM to carry on process management.

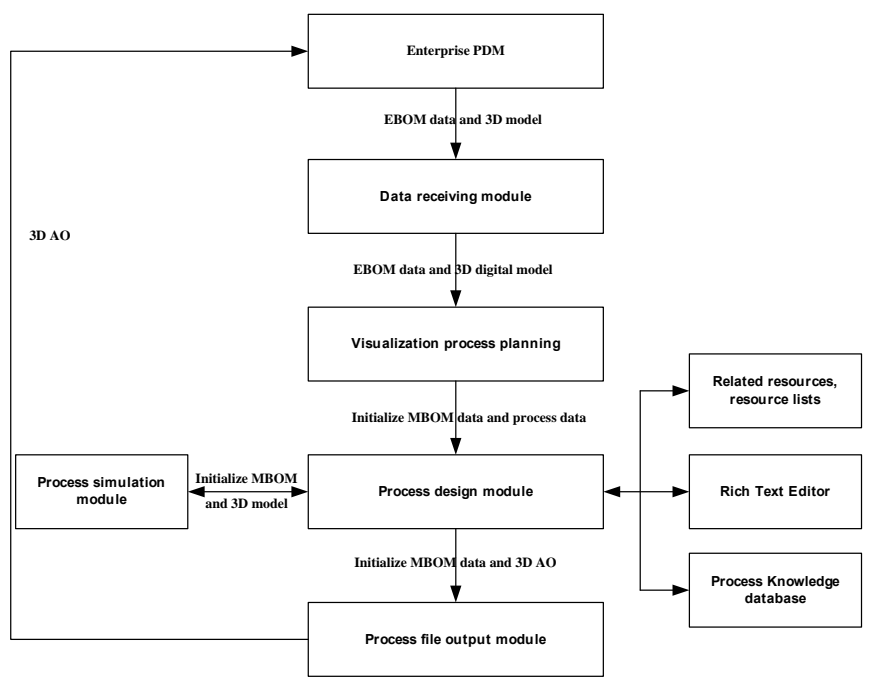

Figure 1. Flowchart of data transferring process

\section{CONVERSION SCHEME OF PPR DATA MODEL}

\subsection{Definition of PPR data model}

Data related to the aircraft assembly process is divided into product, process, resources, and everyone can be represented in hierarchical structure, the hierarchical data model based on the productprocess-resources, referred to as the PPR (Product, Process, Resources) data model. PPR data model is to describe the enterprise in the entire product life cycle, access, sharing and reuse of products, processes, resources, and enterprise knowledge model. For a product object, its manufacturing process includes product information, process knowledge, resources and processes involved, as well as linkages between products, processes and resources, therefore, the PPR data model of a complex product can be expressed as:

$\mathrm{PPR}=\{$ Prod, Proc, Res, Rel $\}$

Where,

Prod: product knowledge of product objects;

Proc: manufacturing processes of products object;

Res: resources of products object used in the manufacturing;

Rel: the relationships among products, processes and resources.

PPR must meet the following relationship:

(1) $\operatorname{Prod} \neq \varnothing$, Proc $\neq \varnothing$, Res $\neq \varnothing$, Rel $\neq \varnothing$;

(2) Prod $\cap$ Proc $=\varnothing$, Prod $\cap$ Res $=\varnothing$.

\subsection{The hierarchical structure of PPR data model}

The hierarchical model is adopted to describe PPR data model. That the linkages between entities are represented by tree structure is called hierarchical model. Many things in the real world are organized hierarchically. Hierarchical data model is proposed, at first, to simulate such things organized hierarchically. In the next section, a review of the representation of hierarchical model is given in detail.

The tree nodes represent entity set (record type), the link line between nodes is the connection between the two entities sets, this relationship can only be "1-M". The entity 1 , usually setting on the top, is called the parent nodes, while the entity $\mathrm{M}$, setting on the bottom, is called child nodes. Hierarchical structure model is characterized by: (1) there is only one root node; (2) other than the root node has one and only one parent node. So hierarchical model can only be said that "1-M" relationship, and not directly said "M-M" relationship. In a hierarchical model, a node, called a record type, is used to describe the entity set. Each record type can have one or more record valuesAn upper record type may correspond to one or more lower records type, while the lower record type can only correspond to one upper record type.

Hierarchy model consistent with assembly relationship of product, the assembly process information is closely product information, and resource information is combined with the hierarchical structure too. This article only gives the hierarchy structure of product, the other hierarchy and so on. Figure 2 is the relationship between the layers of the product assembly. Hierarchical assembly model can completely describe the product assembly information. It not only describes the components of information itself, but also describes the relationship between components and the topology of the assembly; it not only completely describes products information, but also describes the inheritance relationship between product design parameters and constraints of its transformation mechanism.

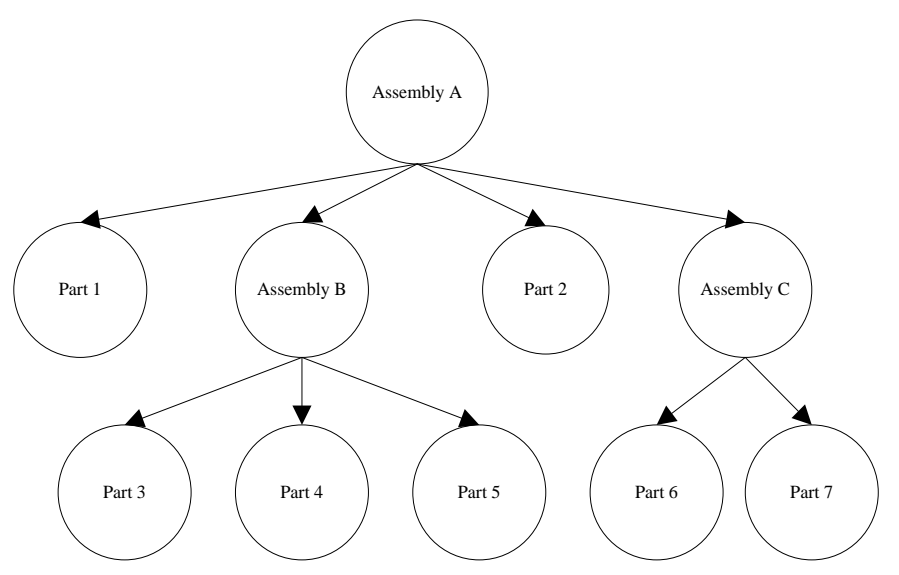

Figure 2. Product hierarchical tree structure diagram

\subsection{XML-Based PPR data model expression}

In our digital assembly process system, the product information is usually expressed in the format of hierarchical tree. Since each product object 
represents an independent assembly member and is the abstract of the specific parts, taking into account the diversity of product objects and attributes of the product tree structure, using hierarchical tree structure to express the structure of the product can well satisfy the demand. Through this structure, the product object of all relevant information can be more efficiently organized. To Figure 2, Figure 3 is diagram as the corresponding table in the format of EBOM tree diagram.

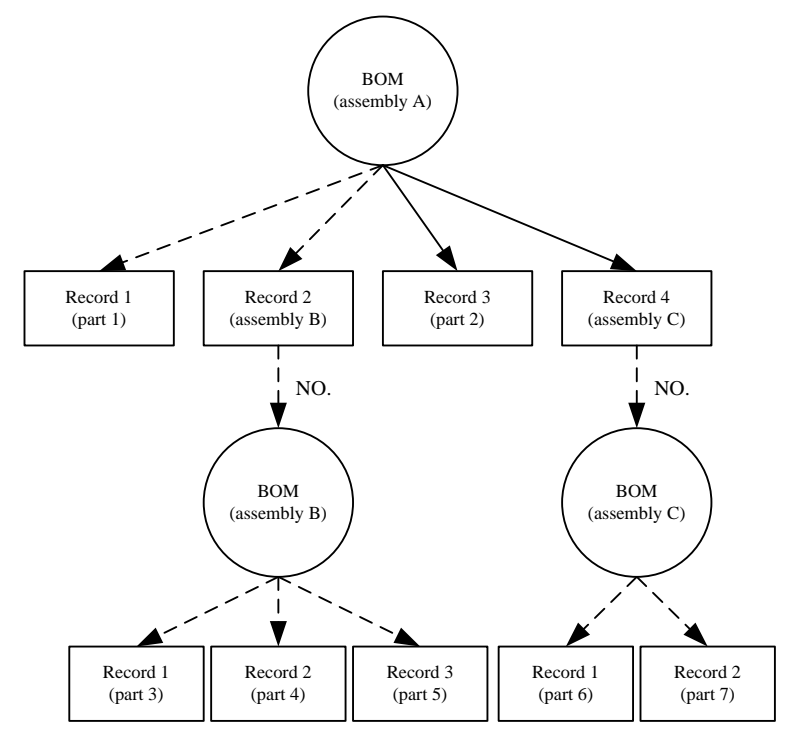

Figure 3. Data structure diagram in style of EBOM table

Product model established by using XML language and combining with multi-level objectoriented way of thinking, to some extent, can fully describe the product information of the aircraft. At the same time, XML language is easy to expand and universal to use, and it is very suitable for information exchange between heterogeneous systems, so our system takes it as the middle media of data exchange between systems. Just as Figure 4 shown, an assembly is expressed by an XML file,

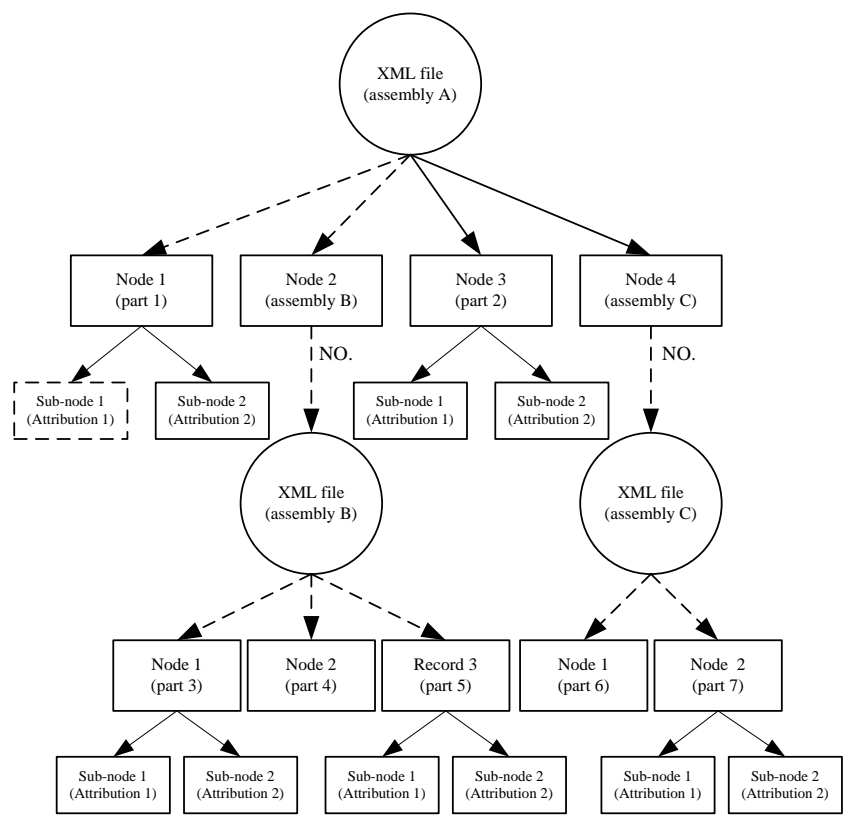

Figure 4. Product information data structure based on XML which is named as the number of assemblies. Assembly parts are expressed by child node under the XML root node, the property of component parts is expressed by child node under component parts child nodes, each relationship between XML file is correlated by the number of assemblies. The instance structure of XML product data model is easy to expand, it can be dynamically configured, each branch is independent, it is easy to modify and edit, in parallel, it is exactly in conformity with the hierarchy structure data model of digital products in the assembly process system.

\subsection{Integration method with PDM system}

The data in the digital assembly process system is divided into product, process and resources. On the basis of three parts, according to the different requirements of enterprises or projects, corresponding template is customized as PTS, which determines the template data attribution, hierarchy relationship, displays structure and other varieties of information. This paper only takes the data receiver from PDM to EBOM as an example, takes the definition of the product data model and its automatic conversion as the description, and the other data processing in the assembly process and so on.

Enterprise data is managed by the PDM system, therefore, the digital process system must integrate PDM to obtain the data, and to get BOM data from the PDM. In this paper, the integration between the application systems is indirect, that is, data transmission between PDM and DPE is completed by the middle file: BOM table in XML format. During the course of data transferring, sending messages between application systems is completed by Soap protocol, therefore, the integrated approach between heterogeneous systems is the system integration based on XML and Soap protocol. There are 4 steps as shown in Figure 5.

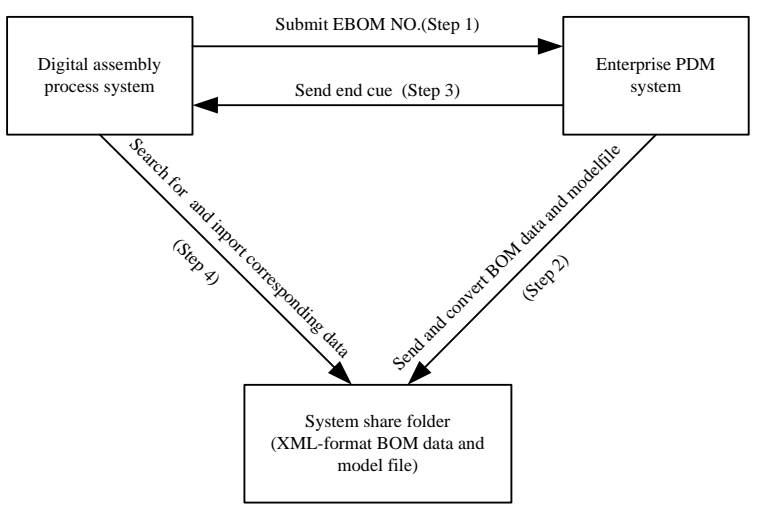

Figure 5. Integration diagram between digital assembly process system and PDM system

Step 1: Digital assembly process system sends a request to the PDM system, and passes the number of EBOM which user import to the PDM system, and then waits for PDM system response. 
Step 2: Once PDM system receives the request, immediately turns the number of EBOM and subEBOM data into a common XML format EBOM data which is send in a shared folder on the system.

Step 3: After the data transmission is finished, PDM system will send "Data transmission complete" message to digital assembly process system.

Step 4: After the digital assembly process System receives "Data transmission complete" message, it will find the corresponding EBOM data and subEBOM data and associated model file in the share folder designated by system, and then bulk import the data.

\section{AN EXAMPLE}

The system is taken the assembly process design of horizontal stabilizer components of one aircraft as an example to illustrate. In the new assembly process design project, enter the DPE first module, select the predefined data model (PTS templates), and then build the project, and enter the interface of the project PPR Navigator which have three nodes: new Product (product), Process (Process) Resources (resources). Just as Figure 6 shows, the interface of import EBOM and establishment of product data. The other work interface will not repeat here.
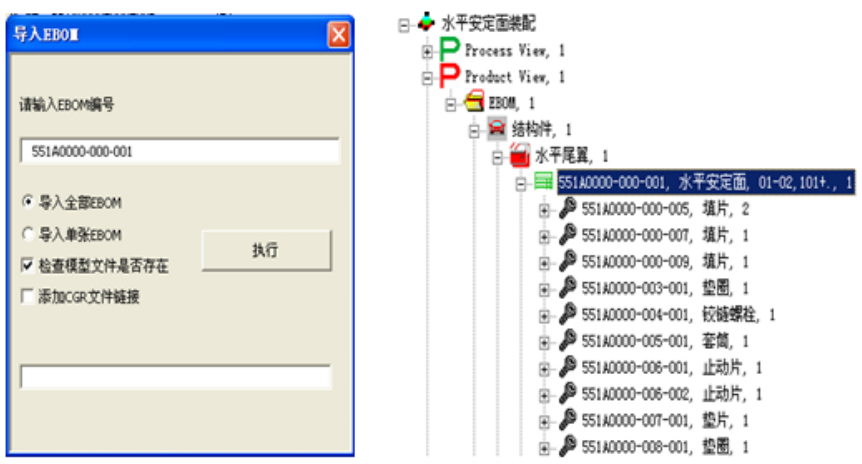

Figure 6. Interface of importing EBOM

\section{CONCLUSION}

In this paper, centering on aircraft digital assembly process system, we in-depth studied the data conversion and integration method of aircraft assembly process system, we mainly do the following works.

(1)To transfer model data from CAD system to integrated virtual assembly environment, we put forward information extraction \& model conversion method. In this method, the process of assembly model transformation was divided into two processes, information extraction and model conversion. Model data information was transferred into the VE by secondary development of CAD system and model conversion. The application indicated that the method was feasible and the effectiveness of data transformation was validated.

(2)According to the characteristics of the aircraft assembly process, the hierarchical PPR data model of aircraft assembly process is defined. Based on XML, the conversion among various types of aircraft assembly process data model are implemented, the basis for following tasks of digital assembly of aircraft is provided.

(3)We developed the assembly process system of aircraft, and the system can be integrated with PDM system. At Last, an example of assembly on the components of aircraft's nose was given, and the result was proved to be effective.

\section{ACKNOWLEDGMENTS}

The project is supported by the Science and Technology Program of Shandong Provincial Education Department, (Grant No. J12LB69), partially supported by the Project of Linyi 2014 Science and Technology Program (Grant No. 201414028).

\section{REFERENCES}

[1] JAYARAMS, JAYARAMU, WANGY. 1999. VADE: a virtual assembly de-sign environment. Journal of Computer Graphics and Applications, 19 (6): 44-50.

[2] WAN Huagen, GAO Shuming, PENG Qunsheng. 2002. VDVAS: an integrated virtual design and virtual assembly environment. Chinese Journal of Computer \& Graphics, 7(1): 27-35.

[3] Liberman LI, M A Wesley. 1979. AUTOPASS: An automatic program-ming system for computer controlled mechanical assembly. IBM Journal of Research and Development, 21(4): 321-333.

[4] Kunwoo Lee, David C Gossard. 1985. A hierarchical data structure for representing assemblies.Part. Journal of.Computer-Aided Design, 17(1): 15-19

[5] ZHU Wen-feng, LAIXin-min, LIN Zhong-qin, WANG Hao. 2005. Research on virtual prototyping modeling for auto-body assembly. Journal of Computer Integrated Manufacturing Systems. 11(1): 63-67.

[6] ZHANG Lian-dou, WU Yan-peng, ZHAO Huan, ZHANG Jian-min. 2005. Design and Implementation of Hierarchical-Tree Assembly Data Model Based on Object Oriented. Journal of Computer Simulation. 22(2): 133-135.

[7] WANG Cheng-en, YU Hong, ZHANG Wen-lei, YU Jiapeng. 2010. Object-oriented aero-engine assembly models, Journal of Computer Integrated Manufacturing Systems. 16(5):942-948.

[8] ZHANG Gang, YIN Guo-fu, DENG Ke-wen, LI Huosheng. Research on assembly-oriented feature-hierarchy modeling method, journal of Computer Integrated Manufacturing Systems. 11(7)(2005)916-920.

[9] WU Dian-liang, YANG Run-dang, MA Deng-zhe, FAN Xiumin. 2005. Product information representation in integrated virtual assembly environment, Journal of Computer Integrated Manufacturing Systems, 10 (11):1364-1369. 